\title{
Duality between the two-locus Wright-Fisher Diffusion Model and the Ancestral Process with Recombination
}

\author{
Shuhei Mano \\ The Institute of Statistical Mathematics, \\ Tachikawa 190-8562, Japan \\ and \\ Japan Science and Technology Agency, \\ Kawaguchi 332-0012, Japan
}

\begin{abstract}
Address for correspondence: The Institute of Statistical Mathematics, 10-3 Midori-cho, Tachikawa, Tokyo 190-8562, Japan; Email: smano@ism.ac.jp
\end{abstract}




\begin{abstract}
Known results on the moments of the distribution generated by the two-locus WrightFisher diffusion model and a duality between the diffusion process and the ancestral process with recombination are briefly summarized. A numerical methods for computing moments by a Markov chain Monte Carlo and a method to compute closed-form expressions of the moments are presented. By using the duality argument properties of the ancestral recombination graph are studied in terms of the moments.
\end{abstract}

Keywords: duality, diffusion process, ancestral graph, population genetics, recombination 


\section{INTRODUCTION}

In classical population genetics theory behavior of frequency of a gene type (allele) has been a central issue (for example, 2]). The fate of the allele frequency has been modeled by a diffusion process, where the population size is assumed to be sufficiently large. The diffusive limit, which is called as the Wright-Fisher diffusion model, is expected to illustrate actual evolution of the allele frequency in the population. Numerical methods for computing likelihood of a sample taken from the equilibrium have attracted much interest (for example, [22]). Explicit and closed-form expressions of the whole process have importance by their own right. Unfortunately, their availability has been limited. For the one-locus two-allele model without mutation and other evolutionary forces closedform expressions of the probability density of the allele frequency at a fixed time were obtained in terms of orthogonal polynomials [16],[10]. In contrast, for two-locus models known closed-form expressions have been limited to several moments of the distribution generated by the diffusion process [20], 15]. A comprehensive survey at early 1970's, which is still useful, is [15]. Recently, closed-form expressions of a class of moments were obtained in terms of orthogonal polynomials [17].

The concept of duality has been a powerful tool in stochastic analysis of interacting particle systems [14]. In population genetics theory the moment dual of the Wright-Fisher diffusion model was firstly used in [21]. A genealogical process of a sample taken from a population, which is known as the coalescent [12], has been useful for population genetic data analyses. The duality was used to obtain branching-coalescent processes as models of natural selection [13] and conversion bias [19]. Ancestral processes are process of numbers of ancestral lineages in a section of ancestral graphs, which are analogues of the coalescent genealogy. The dual of the one-locus two-allele model Wright-Fisher diffusion model with directional selection [1] is an ancestral process, which is the number of ancestral lineages in a section of the ancestral selection graph. The dual is a birth and death process with linear birth and quadratic death rates. It was demonstrated that properties of the birth 
and death process can be studied by referring to classical results on the Wright-Fisher diffusion model [18]. For the multi-locus model an analogue of the coalescent genealogy, called the ancestral recombination graph (ARG), was introduced [7]. The two-locus ARG integrates marginal genealogies at the two loci. The ancestral process, which is a process of the numbers of ancestral lineages in a section of the ARG, is the dual of the two-locus two-allele Wright-Fisher diffusion model [5].

In section 2, we briefly summarize known results on the moments of the distribution generated by the two-locus two-allele Wright-Fisher diffusion model. In section 3, the moment duality between the diffusion process and the ancestral process, which is a process of the numbers of ancestral lineages in a section of an ARG, is introduced. A numerical method for computing moments at a fixed time by a Markov chain Monte Carlo is introduced. In section 4, method to compute closed-form expressions of the moments by using an ARG terminology is presented. In section 5, by using the duality argument properties of the ARG are studied in terms of the moments.

\section{Summary OF KNOWN RESUlts ON THE MOMENTS}

Consider a random mating monoecious diploid population consisting of $N$ individuals. Two linked loci $A$ and $B$ are segregating, where recombination fraction between the two loci is $r$. Pairs of alleles $A_{1}, A_{2}$ and $B_{1}, B_{2}$ are in the loci $A$ and $B$, respectively. A diffusive limit is measuring time in units of $2 N$ generations and $2 N \rightarrow \infty$, while $\rho=4 N r$ is kept constant. Let frequencies of gametes $A_{1} B_{1}, A_{1} B_{2}, A_{2} B_{1}$, and $A_{2} B_{2}$, be respectively, $x_{1}$, $x_{2}, x_{3}$, and $1-x_{1}-x_{2}-x_{3}$. Frequencies of the alleles $A_{1}$ and $A_{2}$ are denoted by $x$ and $1-x$, respectively, and those of the alleles $B_{1}$ and $B_{2}$ are denoted by $y$ and $1-y$, respectively. Then $x=x_{1}+x_{2}$ and $y=x_{1}+x_{3}$. Set $z=x_{1}\left(1-x_{1}-x_{2}-x_{3}\right)-x_{2} x_{3}$, which is a measure of association between $x$ and $y$. The limiting diffusion process $\left\{x_{1}(t), x_{2}(t), x_{3}(t) ; t \geq 0\right\}$ is defined in a simplex

$$
K: 0 \leq x_{1} \leq x_{1}+x_{2} \leq x_{1}+x_{2}+x_{3} \leq 1
$$


Let $H=\Phi(K)$, where $\Phi\left(x_{1}, x_{2}, x_{3}\right)=(x, y, z)$ is a $C^{\infty}$-diffeomorphism of $K$ onto $H$. The generator of the diffusion process $\{x(t), y(t), z(t) ; t \geq 0\}$ in $H$ is [20]

$$
\begin{aligned}
\mathcal{L}= & \frac{x(1-x)}{2} \frac{\partial^{2}}{\partial x^{2}}+\frac{y(1-y)}{2} \frac{\partial^{2}}{\partial y^{2}}+z \frac{\partial^{2}}{\partial x \partial y}+z(1-2 x) \frac{\partial^{2}}{\partial x \partial z}+z(1-2 y) \frac{\partial^{2}}{\partial y \partial z} \\
& -z\left(1+\frac{\rho}{2}\right) \frac{\partial}{\partial z}+\frac{1}{2}\left\{x y(1-x)(1-y)+z(1-2 x)(1-2 y)-z^{2}\right\} \frac{\partial^{2}}{\partial z^{2}}
\end{aligned}
$$

In the classical population genetics theory some problems of general interests are concerning events of fixation. The probability of eventual fixation of an allele and the probability density of the time to the fixation have been studied. Some of these properties can be studied in terms of moments of the distribution generated by the model by using moment inversion formula. The probability of eventual fixation of a gamete in the two-locus two-allele Wright-Fisher diffusion model governed by the generator (2.1) is obtained immediately by the moments. In fact, since the stationary density is atomic, $\lim _{t \rightarrow \infty} \mathbb{E}[x(t) y(t)]$ gives the fixation probability of the gamete $A B$. For an allele two types of fixation can be defined; first fixation occurs when the first of the four alleles is lost and the final fixation occurs when an allele at the other locus is lost (a gamete fix). These fixation times are

$$
\begin{aligned}
& T_{1}=\inf \{t \geq 0 ; x(t)(1-x(t)) y(t)(1-y(t))=0\}, \\
& T_{0}=\inf \{t \geq 0 ; x(t)(1-x(t))+y(t)(1-y(t))=0\},
\end{aligned}
$$

respectively. The probability densities are

$$
\begin{aligned}
& \mathbb{P}\left[T_{1}<t\right]=\lim _{n \rightarrow \infty} \mathbb{E}\left[\{1-x(t)(1-x(t)) y(y)(1-y(t))\}^{n}\right], \\
& \mathbb{P}\left[T_{0}<t\right]=\lim _{n \rightarrow \infty} \mathbb{E}\left[\{1-x(t)(1-x(t))-y(y)(1-y(t))\}^{n}\right],
\end{aligned}
$$

respectively. It seems impossible to obtain explicit and closed-form expressions of these limits (see Section 4). Nevertheless, some of the moments whose closed-form expressions are available are useful to obtain upper bounds and approximate formula of these probabilities [15]. By the same reason a closed-form expression of the joint distribution of $(x(t), y(t), z(t))$ at a fixed time $t$ is not available. 
Let us introduce a classification of the moments of the distribution generated by the generator (2.1).

Definition 2.1. The rank and class of a moment, which is an expectation of a monomial $x^{l} u^{m} x_{1}^{n}, l, m, n \in \mathbb{Z}_{+}$, are $l+m+2 n$ and $n+\min \{l, m\}$, respectively. The rank is equals to or larger than twice of the class.

Remark 2.2. The class-zero moments have closed-form expressions and they are the moments of the one-locus Wright-Fisher diffusion model [10]. The class-one moments have closed-form expressions [17] (see below).

Other moments whose closed-form expressions have been obtained are expectations of a type of polynomials:

Lemma 2.3 (Lemma 3.6.1 of [15]). The manifold of polynomials spanned by the set of polynomials $\left\{x^{l}(1-x)^{l} y^{m}(1-y)^{m} z^{n}(1-2 x)^{a}(1-2 y)^{a}\right\}$, where $a=0,1$ and $l, m>0$ if $n=0$, is closed under the operation of $\mathcal{L}$.

Remark 2.4. The polynomials have zero on the boundary of the square $x(1-x) y(1-y)=0$ and $z=0$. Known closed-form expressions for the moments of polynomials of this type are $(l, m, n, a)=(1,1,0,0),(0,0,2,0)$, and $(0,0,0,1)$ by [20]. Expressions for $(2,1,0,0)$, $(1,2,0,0),(2,0,1,1),(0,2,1,1),(2,0,2,0),(0,2,2,0),(2,2,0,0),(1,1,1,1),(1,1,2,0)$, $(0,0,3,1)$, and $(0,0,4,0)$ are given by [15], where the expressions involve eigenvalues whose closed-form expressions are not available.

In [17] a closed-form expression of $\mathbb{E}\left[x_{1}(t) \mid x(t) \in(0,1)\right]$ was obtained by using a limit of a closed-form expression of a class-one moment. It yields an expression of the conditional covariance between $x$ and $y$ given that alleles $A_{1}$ and $A_{2}$ are segregating in the locus $A$. This expression has importance in interpreting observable polymorphism in population genetic data analysis. Here, we summarize some results in [17], because the explicit 
expressions are used in the later sections. If the argument equals to unity, the truncated hypergeometric series

$$
y_{n}(a, b ; c ; z)=\sum_{i=0}^{n} \frac{(a)_{i}(b)_{i}}{(c)_{i} i !} z^{i}
$$

is expressed by the generalized hypergeometric series [1]

$$
y_{n}(a, b, c ; 1)=\frac{\Gamma(a+n+1) \Gamma(b+n+1)}{n ! \Gamma(a+b+n+1)}{ }_{3} F_{2}(a, b, c+n ; c, a+b+n+1 ; 1) .
$$

where ${ }_{3} F_{2}(\cdot)$ is the generalized hypergeometric series. A trivial but useful identity is

Lemma 2.5 ([17]). For $m, n \in \mathbb{Z}_{+}$and $a, b, c \in \mathbb{C}$,

$$
\begin{aligned}
& \frac{n ! \Gamma(a+b+n+1)}{\Gamma(a+n+1) \Gamma(b+n+1)} y_{n}(a, b ; a+b+m+1 ; 1) \\
& =\frac{m ! \Gamma(a+b+m+1)}{\Gamma(a+m+1) \Gamma(b+m+1)} y_{m}(a, b ; a+b+n+1 ; 1) .
\end{aligned}
$$

Remark 2.6. If $m=0$, it gives an identity

$$
\begin{aligned}
{ }_{2} F_{1}(a, b ; a+b+1 ; 1) & ={ }_{3} F_{2}(a, b, a+b+n+1 ; a+b+1, a+b+n+1 ; 1) \\
& =\frac{n ! \Gamma(a+b+n+1)}{\Gamma(a+n+1) \Gamma(b+n+1)} y_{n}(a, b, a+b+1 ; 1) \\
& =\frac{\Gamma(a+b+1)}{\Gamma(a+1) \Gamma(b+1)},
\end{aligned}
$$

which is a spacial case of the Gauss hypergeometric theorem [1].

An expression of a power of $p$ in terms of the Gegenbauer polynomial follows by the orthogonal and complete property [17]:

$$
p^{n}=\sum_{m=2}^{n+2} 2(2 m-1) \frac{[n+1]_{m-1}}{(n+1)_{m+1}}(-1)^{m} T_{m-2}^{1}(1-2 p), \quad n \in \mathbb{Z}_{+},
$$

where $T_{m}^{1}(\cdot)$ is the Gegenbauer polynomial, which also denoted by $C_{1}^{\left(\frac{3}{2}\right)}(\cdot) . \quad[n]_{m}$ and $(n)_{m}$ are falling and rising factorials, respectively. By using this expression closed form solutions of systems of differential equations for class-one moments were obtained. Let $\mu_{l, m, n}(t)=\mathbb{E}_{p q d}\left[x(t)^{l} y(t)^{m} z(t)^{n}\right]$ for $l, m, n \in \mathbb{Z}_{+}$. 
Proposition $2.7(([17]))$. For $n \in \mathbb{Z}_{+}$,

$$
\mu_{n, 0,1}(t)=\sum_{m=2}^{n+2} 2(2 m-1) \frac{[n+1]_{m-1}}{(n+1)_{m+1}}(-1)^{m} T_{m-2}^{1}(1-2 p) d e^{-\frac{m(m-1)+\rho}{2} t} .
$$

Proposition 2.8 ([17]). For $n \in \mathbb{Z}_{+}$,

$$
\mu_{n, 1,0}(t)=p q+\frac{2 d}{2+\rho}+\sum_{m=1}^{n-1} E_{n}^{(m)} e^{-\frac{m(m+1)}{2} t}+\sum_{m=1}^{n} F_{n}^{(m)} e^{-\frac{\rho+m(m+1)}{2} t}
$$

except for $\rho=(k+m)(k-m-1), k=m+2, m+3, \ldots, n ; m=1,2, \ldots, n$, where

$$
\begin{aligned}
E_{n}^{(m)}= & (-1)^{m} \frac{[n]_{m+1}}{(n)_{m+1}}\left[\frac{2(2 m+1)}{m(m+1)} p(1-p) q T_{m-1}^{1}(1-2 p)\right. \\
& \left.+2\left\{\frac{T_{m}^{1}(1-2 p)}{2(m+1)+\rho}+\frac{T_{m-2}^{1}(1-2 p)}{2 m-\rho}\right\} d\right]
\end{aligned}
$$

and

$$
F_{n}^{(m)}=2(-1)^{m} \frac{[n]_{m}}{(n)_{m}}\left\{\frac{1}{2 m+\rho}+\frac{1}{2(m+1)-\rho} \frac{(n-m)(n-m-1)}{(n+m)(n+m+1)}\right\} T_{m-1}^{1}(1-2 p) d,
$$

with conventions that the first sum is zero if $n=1$ and $T_{-1}^{1}(\cdot)=0$.

Proof. A sketch of the proof was given in [17]. A system of differential equations for the moments gives

$$
E_{n}^{(m)}=\frac{[n]_{m+1} !(2 m+1) !}{(n)_{m+1}(m+1) ! m !} E_{m+1}^{(m)}, \quad n \in \mathbb{Z}_{+} ; m=1,2, \ldots, n-1,
$$

and

$$
\begin{aligned}
&\{(n+m)(n-m-1)-\rho\} F_{n}^{(m)}=4 n(2 m+1) \frac{[n-1]_{m-1}}{(n+1)_{m+1}}(-1)^{m+1} T_{m-1}^{1}(1-2 p) d \\
&(2.5) \quad+n(n-1) F_{n-1}^{(m)}, \quad n \in \mathbb{Z}_{+} ; m=1,2, \ldots, n,
\end{aligned}
$$

with the initial condition

$$
p^{n} q=p q+\frac{2 d}{2+\rho}+\sum_{m=1}^{n-1} E_{n}^{(m)}+\sum_{m=1}^{n} F_{n}^{(m)}, \quad n \in \mathbb{Z}_{+} .
$$

By using (2.2) with $m=1,2$, it is straightforward to solve (2.5) for $F_{n}^{(m)}$ and the solution is (2.4), except for $\rho=(k+m)(k-m-1), k=m+2, m+3, \ldots, n ; m=1,2, \ldots, n$ (The 
exceptional values given in [17] are incorrect). By setting $E_{n}^{(m)}=q E_{n, 1}^{(m)}(p)+d E_{n, 2}^{(m)}(p)$, the initial condition gives

$$
\begin{aligned}
\sum_{m=1}^{n-1} E_{n, 1}^{(m)}(p)= & p(p-1) \sum_{i=0}^{n-2} p^{i} \\
= & p(p-1) \sum_{i=0}^{n-2} \sum_{m=2}^{i+2} 2(2 m-1) \frac{[i+1]_{m-1}}{(i+1)_{m+1}}(-1)^{m} T_{m-2}^{1}(1-2 p) \\
= & p(p-1) \sum_{m=1}^{n-1} 2(-1)^{m+1} \frac{m !(m-1) !}{(2 m) !} \\
& \times y_{n-m-1}(m+1, m, 2 m+2 ; 1) T_{m-1}^{1}(1-2 p) \\
= & p(1-p) \sum_{m=1}^{n-1}(-1)^{m} \frac{[n]_{m+1}}{(n)_{m+1}} \frac{2(2 m+1)}{m(m+1)} T_{m-1}^{1}(1-2 p),
\end{aligned}
$$

for each $n=2,3, \ldots$, where the second equality holds by (2.3) and the last equality holds by (2.2) with $m=0$. The expression of summation of $E_{n, 2}^{(m)}(p)$ follows by re-arrangement of terms.

\section{The DuAlity And A nUmerical Method FOR COMPUTING MOMENTS}

The process of the numbers of lineages including non-ancestral lineages (see below) in a section of a two-locus ARG is a birth and death process [7]. When there are $i$ lineages, the birth rate is $i \rho / 2$ and the death rate is $i(i-1) / 2$. The birth and death process is identical to the numbers of ancestral lineages in a section of an ancestral selection graph, and the moment dual of the birth and death process is the Wright-Fisher diffusion of the one-locus two-allele model with directional selection [18]. Note that, an ARG involves gametes whose alleles are not ancestral to any allele in a sample. We denote alleles which are not ancestral to any allele in the sample by -, since in principle the allelic state of the locus cannot be specified by the sample. For example, a gamete $A B$ could be a recombinant descendant of $A-$, which in turn could be a recombinant descendant of -- . The gamete -- is involved in the ARG, nevertheless, whose alleles are not ancestral to any allele in the sample. In this paper, we discuss the ancestral process which generates 
numbers of ancestral lineages in a section of an ARG. The ancestral lineages are a subset of lineages in an ARG. The stationary distribution of the process with infinitely-manyallele mutation model was studied by [6]. A whole graph $\mathcal{G}$ includes marginal genealogies $\mathcal{T}_{A}$ and $\mathcal{T}_{B}$ at the locus $A$ and $B$, respectively. Denote the edges of a graph by $E(\cdot)$. Edges of $\mathcal{G}$ are partitioned into $\mathcal{A}=E(\mathcal{G}) \cap E\left(\mathcal{T}_{A}\right) \cap E\left(\mathcal{T}_{B}\right)^{c}, \mathcal{B}=E(\mathcal{G}) \cap E\left(\mathcal{T}_{A}\right)^{c} \cap E\left(\mathcal{T}_{B}\right)$, $\mathcal{C}=E(\mathcal{G}) \cap E\left(\mathcal{T}_{A}\right) \cap E\left(\mathcal{T}_{B}\right)$ and $\mathcal{D}=E(\mathcal{G}) \cap E\left(\mathcal{T}_{A}\right)^{c} \cap E\left(\mathcal{T}_{B}\right)^{c}$. We call $\mathcal{A}, \mathcal{B}$ and $\mathcal{C}$ ancestral lineages. $\mathcal{D}$ are not ancestral lineages since they are not ancestral to any allele in a sample. Let $\mathcal{E}_{t}$ be the edges of a section of $\mathcal{G}$ taken at time $t$ backwards. Denote the number of ancestral lineages by $a(t)=\left|\mathcal{E}_{t} \cap \mathcal{A}\right|, b(t)=\left|\mathcal{E}_{t} \cap \mathcal{B}\right|, c(t)=\left|\mathcal{E}_{t} \cap \mathcal{C}\right|$. The marginal transition rates of $(a(t), b(t), c(t))$ do not depends on $\left|\mathcal{E}_{t} \cap \mathcal{D}\right|$ and is Markovian [5, 7]. The rates are:

$$
(a, b, c) \rightarrow \begin{cases}(a+1, b+1, c-1), & c \rho / 2, \\ (a-1, b-1, c+1), & a b, \\ (a-1, b, c), & a c+a(a-1) / 2, \\ (a, b-1, c), & b c+b(b-1) / 2, \\ (a, b, c-1), & c(c-1) / 2,\end{cases}
$$

with $a+b+c>1$. The backward equation for the joint probability generating function $\xi_{l, m, n}(t)=\mathbb{E}_{l, m, n}\left[p^{a(t)} q^{b(t)} g^{c(t)}\right]$ of the Markov chain $\{a(t), b(t), c(t) ; t \geq 0\}$ on the integer lattice $\mathbb{Z}_{+}^{3} \backslash \mathbf{0}$ is

$$
\begin{aligned}
\frac{d \xi_{l, m, n}}{d t}= & -\frac{(l+m+n)(l+m+n-1)+n \rho}{2} \xi_{l, m, n}+\frac{n \rho}{2} \xi_{l+1, m+1, n-1} \\
& +\frac{l(l-1+2 n)}{2} \xi_{l-1, m, n}+\frac{m(m-1+2 n)}{2} \xi_{l, m-1, n}+\frac{n(n-1)}{2} \xi_{l, m, n-1} \\
& +l m \xi_{l-1, m-1, n+1},
\end{aligned}
$$

for $(l, m, n) \in \mathbb{Z}_{+}^{3} \backslash \mathbf{0}$, where terms whose subscripts have negative integer are zero. It is straightforward to see that the moments $\nu_{l, m, n}(t)=\mathbb{E}_{p, q, g}\left[x(t)^{l} y(t)^{m} x_{1}(t)^{n}\right]$ also satisfy the system of the differential equations (3.2). Therefore a moment duality follows immediately 
[5]. We give a proof, since it is useful to introduce a numerical method for computing moments.

Lemma 3.1. The diffusion process $\{x(t), y(t), z(t) ; t \geq 0\}$ in $H$ with $\left(x(0), y(0), x_{1}(0)\right)=$ $(p, q, g)$ and the Markov chain $\{a(t), b(t), c(t) ; t \geq 0\}$ in $\mathbb{Z}_{+}^{3} \backslash \mathbf{0}$ whose transition rates are (3.1) with $(a(0), b(0), c(0))=(l, m, n)$ are dual to each other:

$$
\mathbb{E}_{p, q, g}\left[x(t)^{l} y(t)^{m} x_{1}(t)^{n}\right]=\mathbb{E}_{l, m, n}\left[p^{a(t)} q^{b(t)} g^{c(t)}\right]
$$

Proof. The system of the differential equations (3.2) is equivalent to an integro-recurrence equation

$$
\xi_{l, m, n}(t)=\int_{0}^{t} \mathcal{T} \xi_{l, m, n}(s) e^{-\gamma_{l, m, n}(t-s)} d s, \quad l, m, n \in \mathbb{Z}_{+},
$$

where

$$
\begin{aligned}
\mathcal{T} \xi_{l, m, n}= & \frac{n \rho}{2} \xi_{l+1, m+1, n-1}+\frac{l(l-1+2 n)}{2} \xi_{l-1, m, n}+\frac{m(m-1+2 n)}{2} \xi_{l, m-1, n} \\
& +\frac{n(n-1)}{2} \xi_{l, m, n-1}+l m \xi_{l-1, m-1, n+1}
\end{aligned}
$$

and $\gamma_{l, m, n}=((l+m+n)(l+m+n-1)+n \rho) / 2$. The integro-recurrence equation is recast into

$$
\xi_{l, m, n}(t)=\int_{0}^{t} \sum_{l^{\prime}, m^{\prime}, n^{\prime}} \mathbb{P}\left[l^{\prime} m^{\prime} n^{\prime} \mid l, m, n\right] \xi_{l^{\prime}, m^{\prime}, n^{\prime}}(t-s) \gamma_{l, m, n} e^{-\gamma_{l, m, n} s} d s,
$$

where the transition probability is given by dividing the rates in (3.1) by $\gamma_{a, b, c}$. Meanwhile

$$
\begin{aligned}
\mathbb{E}_{l, m, n}\left[p^{a(t)} q^{b(t)} g^{c(t)}\right] & =\mathbb{E}_{l, m, n}\left\{\mathbb{E}\left[p^{a(t)} q^{b(t)} g^{c(t)} \mid(a(s), b(s), c(s))=\left(l^{\prime}, m^{\prime}, n^{\prime}\right)\right]\right\} \\
& =\mathbb{E}_{l, m, n}\left\{\mathbb{E}_{l^{\prime}, m^{\prime}, n^{\prime}}\left[p^{a(t-s)} q^{b(t-s)} g^{c(t-s)}\right]\right\} \\
& =\mathbb{E}_{l, m, n}\left[\xi_{l^{\prime}, m^{\prime}, n^{\prime}}(t-s)\right],
\end{aligned}
$$

where the second equality follows by the strong Markov property. This expression is equivalent to (3.4). Therefore $\xi_{l, m, n}(t)=\mathbb{E}_{l, m, n}\left[p^{a(t)} q^{b(t)} g^{c(t)}\right]$. On the other hand, it is 
straightforward by Itô's formula to see that $\nu_{l, m, n}(t)=\mathbb{E}_{p, q, g}\left[x(t)^{l} y(t)^{m} x(t)^{n}\right]$ satisfy the system of differential equations (3.2).

The duality relation is useful for numerical computation of the moments $\nu_{l, m, n}(t)$ by simulating independent copies of $(a(t), b(t), c(t))$ by the Markov chain Monte Carlo with the transition probabilities (3.1). Consider the simulations stopped at a time $t$. The average over $p^{a(t)} q^{b(t)} g^{c(t)}$ of the copies is then an unbiased estimator of the moment $\nu_{l, m, n}(t)$. A similar method was used for computing likelihood of a sample in a varying environment [8]. The simulation can be stopped before $t$. Let a hitting time be

$$
\tau=\inf \{s \geq 0 ;(a(s), b(s), c(s)) \in \mathcal{S}\}
$$

where $\mathcal{S}=\{(0,0,1),(1,1,0)\}$ is the closed set of states from which a chain cannot exit. If $\tau<t$

$$
\begin{aligned}
\mathbb{E}_{l, m, n}\left[p^{a(t)} q^{b(t)} g^{c(t)}\right]= & \mathbb{E}_{l, m, n}\left\{\mathbb{E}\left[p^{a(t)} q^{b(t)} g^{c(t)} \mid\left(a(\tau), b(\tau), c(\tau)=\left(l^{\prime}, m^{\prime}, n^{\prime}\right)\right]\right\}\right. \\
= & \mathbb{E}_{l, m, n}\left\{\mathbb{E}_{l^{\prime}, m^{\prime}, n^{\prime}}\left[p^{a(t-\tau)} q^{b(t-\tau)} g^{c(t-\tau)}\right]\right\} \\
= & \mathbb{P}_{l, m, n}[(a(\tau), b(\tau), c(\tau))=(0,0,1)] \nu_{0,0,1}(t-\tau) \\
& +\mathbb{P}_{l, m, n}[(a(\tau), b(\tau), c(\tau))=(1,1,0)] \nu_{1,1,0}(t-\tau),
\end{aligned}
$$

where the second equality follows by the strong Markov property, and

$$
\nu_{0,0,1}(t-\tau)=g-\frac{\rho(g-p q)}{2+\rho}\left(1-e^{-\frac{2+\rho}{2}(t-\tau)}\right)
$$

and

$$
\nu_{1,1,0}(t-\tau)=p q+\frac{2(g-p q)}{2+\rho}\left(1-e^{-\frac{2+\rho}{2}(t-\tau)}\right)
$$

From these observations we have a numerical method for computing moments:

Proposition 3.2. Set a Markov time $\sigma=t \wedge \tau$, where $x \wedge y=\min \{x, y\}$. An unbiased estimator of $\nu_{l, m, n}(t)$ is the average over following values obtained by independent copies of 
$(a(\sigma), b(\sigma), c(\sigma))$ simulated by the Markov chain Monte Carlo with the transition probabilities (3.1): if $\sigma=t$, the value is $p^{a(\sigma)} q^{b(\sigma)} g^{c(\sigma)}$. If $\sigma=\tau$ and $(a(\sigma), b(\sigma), c(\sigma))=(0,0,1)$, the value is $\nu_{0,0,1}(t-\tau)$. If $\sigma=\tau$ and $(a(\sigma), b(\sigma), c(\sigma))=(1,1,0)$, the value is $\nu_{1,1,0}(t-\tau)$.

\section{Closed-FORM eXPressions of MOMEnts}

Since the system of differential equations for the moments of the distribution generated by the two-locus two-allele Wright-Fisher diffusion model (3.2) is that for the joint probability generating function of the distribution of the numbers of ancestral lineages in a section of a two-locus ARG, relationship among moments can be specified in terms of events on the ARG. In (3.3), the first event is a recombination, the second and the third events are marginal coalescence in $\mathcal{T}_{A}$ and $\mathcal{T}_{B}$, respectively, and the forth event is a joint coalescence in both of $\mathcal{T}_{A}$ and $\mathcal{T}_{B}$. We call the fifth event as a null coalescence, because coalescence events occur in neither $\mathcal{T}_{A}$ nor $\mathcal{T}_{B}$. The following lemma is obvious.

Lemma 4.1. The manifold of moments spanned by the set of moments whose ranks and classes are equals to or smaller than specified values is closed under the operation of $\mathcal{T}$. Neither of class and rank of a moment change under recombination and null coalescence operations.

It was shown that the moments $\mu_{l, m, n}(t)$ can be obtained recursively from the smaller rank moments [15]. We present a method to compute closed-form expressions of the moments $\nu_{l, m, n}(t)$ by using the ARG terminology, since it gives systematic insights in the computation. In our approach class of a moment is essential. Since closed-form expressions of all moments whose classes are less than two are available (see Section 2), let us start with moments whose classes and ranks are two and $i(\geq 4)$, respectively: $\nu_{i-4,0,2}, \nu_{i-3,1,1}$, $\nu_{i-2,2,0}, \nu_{0, i-4,2}, \nu_{1, i-3,1}$, and $\nu_{2, i-2,0}$. The former three and the latter three moments are closed respectively by recombinations and null coalescences. The expressions for the latter three moments are obtained immediately by exchanging $p$ and $q$ in the expressions of the 
former three moments. The system of three differential equations for the former three moments whose ranks are $j(4 \leq j \leq i)$ are

$$
\begin{aligned}
\frac{d}{d t}\left(\begin{array}{c}
\nu_{j-4,0,2} \\
\nu_{j-3,1,1} \\
\nu_{j-2,2,0}
\end{array}\right)= & \left(\begin{array}{ccc}
-\frac{(j-2)(j-3)+2 \rho}{2} & \rho & 0 \\
(j-3) & -\frac{(j-1)(j-2)+\rho}{2} & \frac{\rho}{2} \\
0 & 2(j-2) & -\frac{j(j-1)}{2}
\end{array}\right)\left(\begin{array}{c}
\nu_{j-4,0,2} \\
\nu_{j-3,1,1} \\
\nu_{j-2,2,0}
\end{array}\right) \\
& +\left(\begin{array}{c}
\frac{(j-1)(j-4)}{2} \nu_{j-5,0,2} \\
\frac{(j-2)(j-3)}{2} \nu_{j-4,1,1} \\
\frac{(j-2)(j-3)}{2} \nu_{j-3,2,0}
\end{array}\right)+\left(\begin{array}{c}
\nu_{j-4,0,1} \\
\nu_{j-3,0,1} \\
\nu_{j-2,1,0}
\end{array}\right),
\end{aligned}
$$

where $\nu_{-1}=0$ by a convention. The solution involves eigenvalues of the matrix in (4.1), which are roots of the cubic equation

$$
\begin{aligned}
& \lambda^{3}+\frac{3 j^{2}-9 j+8+3 \rho}{2} \lambda^{2}+\left\{\frac{3(j-1)^{2}(j-2)^{2}}{2}+\left(3 j^{2}-11 j+15\right) \rho+\rho^{2}\right\} \lambda \\
& +\frac{j(j-1)^{2}(j-2)^{2}(j-3)}{8}+\frac{\left(3 j^{4}-22 j^{3}+65 j^{2}-86 j+48\right) \rho+\left(j^{2}-5 j+8\right) \rho^{2}}{4}=0 .
\end{aligned}
$$

If $j=4$ the system of differential equations (4.1) involves $\nu_{0,0,2}, \nu_{1,1,1}, \nu_{2,2,0}$ and class one moments, we can obtain the closed-form expressions. In fact, the closed-form expressions of the moments whose classes and ranks are two and four, respectively, were obtained by [20]. Solving (4.1) iteratively in $j=5,6, \ldots, i$, we obtain the closed form expressions of the moments whose classes and ranks are two and $i$, respectively.

Computation of the moments whose classes and ranks are $k(\geq 3)$ and $i(\geq 2 k)$, respectively, is in the same manner. To obtain closed-form expressions of these moments a system of $k+1$ differential equations must be solved. The solution involves eigenvalues of a tri-diagonal matrix $A_{k}$ with

$$
\begin{aligned}
& \left(A_{k}\right)_{s, s-1}=(s-1)(j-2 k+s-1) \\
& \left(A_{k}\right)_{s, s}=-\frac{(j-k+s-1)(j-k+s-2)+(k-s+1) \rho}{2} \\
& \left(A_{k}\right)_{s, s+1}=\frac{k-s+1}{2} \rho,
\end{aligned}
$$


for $1 \leq s \leq k+1$ and $2 k \leq j \leq i$. The eigenvalues are roots of the $(k+1)$-th degree algebraic equation. If $k \geq 4$ we cannot expect explicit closed-form expressions of the eigenvalues. The computation eventually involves moments whose classes and ranks are $t$ and $u$, respectively, where $1 \leq t \leq k$ and $i-k+t \leq u \leq 2 t$.

\section{Properties of ARG}

We have been discussed how to compute moments of the distribution generated by the two-locus two-allele Wright-Fisher diffusion model. The moments are useful for studying the two-locus ARG, since the moments of the diffusion, $\nu_{l, m, n}(t)$, is the joint probability generating function of the distribution of the numbers of ancestral lineages in a section of an ARG of a sample $(a(0), b(0), c(0))=(l, m, n)$. We define rank and class of the numbers of ancestral lineages in a section of an ARG, $(l, m, n)$, by $l+m+2 n$ and $n+\min \{l, m\}$, respectively. An ARG of a class-zero sample is a marginal genealogy, whose properties are well known. In the following we consider a sample whose class is larger than zero. Since Lemma 3.1 gives

$$
\lim _{t \rightarrow \infty} \nu_{l, m, n}(t)=\frac{2 g}{2+\rho}+\frac{\rho p q}{2+\rho}, \quad n+\min \{l, m\} \geq 1
$$

the stationary distribution of the numbers of ancestral lineages in a section of an ARG is

$$
\frac{2}{2+\rho} \delta_{(0,0,1)}+\frac{\rho}{2+\rho} \delta_{(1,1,0)}
$$

The distribution of the number of ancestral lineages in a section of an ARG of a sample $(0,0,2)$ can be obtained from the known closed-form expression of the moments of the distribution generated by the two-locus two-allele Wright-Fisher diffusion model [20]. It seems that general formula (applicable to all rank moments) of the distribution of a sample whose class is larger than one are not available. In contrast, we have general formula of the distribution of class-one samples. The closed-form expression can be obtained by using closed-from expressions of the moments with a finite series expansion of the Gegenbauer 
polynomial [3]:

$$
T_{m}^{1}(1-2 p)=\frac{1}{2} \sum_{i=0}^{m} \frac{(-m)_{i}(m+1)_{i+2}}{i !(i+1) !} p^{i} .
$$

Let $\nu_{k, 1,0}(t)=\sum_{l, m, n} f_{l, m, n}(t) p^{l} q^{m} g^{n}$, where $f_{l, m, n}(t)=\mathbb{P}_{k, 1,0}[(a(t), b(t), c(t))=(l, m, n)]$.

The distribution of a sample $(1,1,0)$ is

$$
f_{1,1,0}(t)=\frac{\rho}{2+\rho}+\frac{2}{2+\rho} e^{-\frac{2+\rho}{2} t}, \quad f_{0,0,1}(t)=\frac{2}{2+\rho}\left(1-e^{-\frac{2+\rho}{2} t}\right)
$$

since

$$
\nu_{1,1,0}=p q+\frac{2(g-p q)}{2+\rho}\left(1-e^{-\frac{2+\rho}{2} t}\right) .
$$

For samples $(k, 1,0), k \geq 2$, from Proposition 2.8 and (5.1) the closed-form expressions have general formula. For $i \geq 0$ we have

$$
\begin{aligned}
& f_{i, 0,1}(t)=\frac{2}{2+\rho} \delta_{i, 0}+g_{i, k}(t) \\
& +\sum_{m=i}^{k-1} \frac{(-1)^{m}[k]_{m+1}}{(k)_{m+1} i !(i+1) !}\left[\frac{(-m)_{i}(m+1)_{i+2}}{2(m+1)+\rho}+\frac{(2-m)_{i}(m-1)_{i+2}}{2 m-\rho}\right] e^{-\frac{m(m+1)}{2} t},
\end{aligned}
$$

and for $i \geq 2$ we have

$$
\begin{aligned}
& f_{i, 1,0}(t)=-g_{i-1, k}(t)-\sum_{m=i-1}^{k-1} \frac{(-1)^{m}[k]_{m+1}}{(k)_{m+1}(i-1) ! i !}\left\{(2 m+1)(1-m)_{i-2}(m)_{i}\right. \\
& \left.+\left[\frac{(-m)_{i-1}(m+1)_{i+1}}{2(m+1)+\rho}+\frac{(2-m)_{i-1}(m-1)_{i+1}}{2 m-\rho}\right]\right\} e^{-\frac{m(m+1)}{2} t},
\end{aligned}
$$

and

$$
\begin{aligned}
& f_{1,1,0}(t)=\frac{\rho}{2+\rho}-g_{0, k}(t) \\
& +\sum_{m=1}^{k-1} \frac{(-1)^{m}[k]_{m+1}}{(k)_{m+1}}\left[2 m+1-\frac{(m+1)(m+2)}{2(m+1)+\rho}-\frac{(m-1) m}{2 m-\rho}\right] e^{-\frac{m(m+1)}{2} t},
\end{aligned}
$$

where

$$
\begin{aligned}
g_{i, k}(t)= & \sum_{m=i+1}^{k-1} \frac{(-1)^{m}[k]_{m}}{(k)_{m}}\left[\frac{1}{2 m+\rho}+\frac{1}{2(m+1)-\rho} \frac{(k-m)(k-m-1)}{(k+m)(k+m+1)}\right] \\
& \times \frac{(1-m)_{i}(m)_{i+2}}{i !(i+1) !} e^{-\frac{m(m+1)+\rho}{2} t} .
\end{aligned}
$$


We can obtain closed-form expressions of the distribution of samples a $(k, 0,1), k \geq 1$ in the similar manner.

Let the waiting times until common ancestors of $\mathcal{T}_{A}$ and $\mathcal{T}_{B}$, respectively, be

$$
W_{A}=\inf \{s \geq 0 ; a(s)+c(s)=1\}, \quad W_{B}=\inf \{s \geq 0 ; b(s)+c(s)=1\} .
$$

Proposition 5.1. The waiting time until a sample has common ancestor at both of the two loci is given by

$$
\mathbb{P}_{l, m, n}\left[W_{A} \vee W_{B} \leq t\right]=\mathbb{P}_{l, m, n}[(a(t), b(t), c(t)) \in \mathcal{S}]
$$

where $x \vee y=\max \{x, y\}$. The waiting time until a sample has common ancestor at one of the two loci is given by

$$
\mathbb{P}_{l, m, n}\left[W_{A} \wedge W_{B} \geq t\right]=\sum_{n^{\prime}+\min \left\{l^{\prime}, m^{\prime}\right\} \geq 2} \mathbb{P}_{l, m, n}\left[(a(t), b(t), c(t))=\left(l^{\prime}, m^{\prime}, n^{\prime}\right)\right]
$$

Remark 5.2. A recursion of the expectation of $W_{A} \vee W_{B}$ for the $A R G$ of a sample $(0,0, c)$ is given by Theorem 4 of [7. Theorem 5 of [7] gives a closed-form expression of the joint Laplace transform of $W_{A} \vee W_{B}$ and $W_{A} \wedge W_{B}$ for the $A R G$ of a sample $(0,0,2)$.

The idea of the number of recombination events in a sample was introduced by [9]. The number of recombination events on the two-locus ARG including non-ancestral lineages was considered by [5, 6], and a closed-form expression of the probability generating function of the number of recombination events was given. Here, we consider the number of recombination events on ancestral lineages of an ARG. Let $s(t)$ be the number of recombination events occurring to $\mathcal{C}$ lineages of an ARG in a time interval $(0, t)$. The recombination events are subset of the recombination events occurred on whole lineages of the ARG.

Lemma 5.3. The joint probability generating function of $(a(t), b(t), c(t), s(t))$ is

$$
\mathbb{E}_{l, m, n, 0}\left[p^{a(t)} q^{b(t)} g^{c(t)} v^{s(t)}\right]=\mathbb{E}_{l, m, n, 0}\left[p^{a_{v}(t)} q^{b_{v}(t)} g^{c_{v}(t)} \exp \left\{-\frac{\rho(1-v)}{2} \int_{0}^{t} c_{v}(u) d u\right\}\right]
$$


where $\left\{a_{v}(t), b_{v}(t), c_{v}(t) ; t \geq 0\right\}$ is a modified process of $\{a(t), b(t), c(t) ; t \geq 0\}$ in which the recombination fraction is $r v$, where $0 \leq v \leq 1$.

Proof. Let $\zeta_{l, m, n}(t)=\mathbb{E}_{l, m, n, 0}\left[p^{a(t)} q^{b(t)} g^{c(t)} v^{s(t)}\right]$. For $(l, m, n) \in \mathbb{Z}_{+}^{3} \backslash \mathbf{0}$, we have

$$
\begin{aligned}
\frac{d \zeta_{l, m, n}}{d t}= & -\frac{(l+m+n)(l+m+n-1)+n v \rho}{2} \zeta_{l, m, n}+\frac{n v \rho}{2} \zeta_{l+1, m+1, n-1} \\
& +\frac{l(l-1+2 n)}{2} \zeta_{l-1, m, n}+\frac{m(m-1+2 n)}{2} \zeta_{l, m-1, n}+\frac{n(n-1)}{2} \zeta_{l, m, n-1} \\
& +l m \zeta_{l-1, m-1, n+1}-\frac{n(1-v) \rho}{2} \zeta_{l, m, n}
\end{aligned}
$$

with the initial condition $\xi_{l, m, n}(0)=p^{l} q^{m} g^{n}$. This is uniquely solved by means of the Feynman-Kac formula and the result is Lemma 5.3 .

Theorem 5.4. The conditional probability generating function of the number of $\mathcal{A}$ lineages in a section of an $A R G$ of a sample $(n, 0,1)$ given that no recombination events occur in a time interval $(0, t)$ is

$$
\mathbb{E}_{n, 0,1,0}\left[p^{a(t)} \mid s(t)=0\right]=\tilde{\nu}_{n, 0,1}(t),
$$

where $\tilde{\nu}_{n, 0,1}(t)$ is $\nu_{n, 0,1}(t)$ with setting $q=g=1$ and $\rho=0$.

Proof. By (3.1) we see that $b_{0}(t)=0$ and $c_{0}(t)=1$ for all $t$, and the marginal process $\left\{a_{0}(t) ; t \geq 0\right\}$ is a death process with death rate $i(i+1) / 2$ when $a_{0}(t)=i$. The joint probability generating function of $(a(t), b(t), c(t))$ given that no recombination events occur in a time interval $(0, t)$ is

$$
\begin{aligned}
\lim _{v \rightarrow 0} \mathbb{E}_{n, 0,1,0}\left[p^{a(t)} q^{b(t)} g^{c(t)} v^{s(t)}\right] & =\mathbb{E}_{n, 0,1,0}\left[p^{a(t)} q^{b(t)} g^{c(t)}, s(t)=0\right] \\
& =\mathbb{E}_{n, 0,1,0}\left[p^{a_{0}(t)} q^{b_{0}(t)} g^{c_{0}(t)} \exp \left\{-\frac{\rho}{2} \int_{0}^{t} c_{0}(u) d u\right\}\right] \\
& =g \mathbb{E}_{n, 0,1,0}\left[p^{a_{0}(t)}\right] e^{-\frac{\rho}{2} t},
\end{aligned}
$$

where the first equality follows by Lebesgue's convergence theorem and the second equality follows by Lemma [5.3. Setting $p=q=g=1$, we have $\mathbb{P}_{n, 0,1,0}[s(t)=0]=e^{-\rho t / 2}$, 
while setting $q=g=1$ we have $\mathbb{E}_{n, 0,1,0}\left[p^{a(t)}, s(t)=0\right]=\tilde{\nu}_{n, 0,1}(t) e^{-\frac{\rho}{2} t}$, where $\tilde{\nu}_{n, 0,1}(t)=$ $\mathbb{E}_{n, 0,1,0}\left[p^{a_{0}(t)}\right]$.

Remark 5.5. Explicit closed-form expression of $\tilde{\nu}_{n, 0,1}(t)$ is available in Section 2, since $\nu_{n, 0,1}(t)=\mu_{n+1,1,0}(t)+\mu_{n, 0,1}(t)$. This expression follows immediately by considering the $A R G$. Recombination might occur on the single $\mathcal{C}$ lineage. By Poisson nature of recombination events, the probability that no recombination occur on the single lineage is $e^{-\frac{\rho}{2} t}$. The marginal process $\left\{a_{0}(t) ; t \geq 0\right\}$ follows the death process independently.

Lemma 5.6. Let $\mathcal{S}$ be the absorbing states. The probability generating function of the number of recombination events on ancestral lineages of an ARG until a sample has common ancestor at both of the two loci is

$$
\mathbb{E}_{l, m, n, 0}\left[v^{s(\tau)}\right]=\mathbb{E}_{l, m, n, 0}\left[\exp \left\{-\frac{\rho(1-v)}{2} \int_{0}^{\tau_{v}} c_{v}(u) d u\right\}\right]
$$

where $\tau_{v}=\inf \left\{s \geq 0 ;\left(a_{v}(s), b_{v}(s), c_{v}(s)\right)=\mathcal{S}\right\}$

Proof. Let $\zeta_{l, m, n}=\mathbb{E}_{l, m, n, 0}\left[p^{a(\tau)} q^{b(\tau)} g^{c(\tau)} v^{s(\tau)}\right]$. For $(l, m, n) \in \mathbb{Z}_{+}^{3} \backslash \mathbf{0}$, we have

$$
\begin{aligned}
0= & -\frac{(l+m+n)(l+m+n-1)+n v \rho}{2} \zeta_{l, m, n}+\frac{n v \rho}{2} \zeta_{l+1, m+1, n-1} \\
& +\frac{l(l-1+2 n)}{2} \zeta_{l-1, m, n}+\frac{m(m-1+2 n)}{2} \zeta_{l, m-1, n}+\frac{n(n-1)}{2} \zeta_{l, m, n-1} \\
& +l m \zeta_{l-1, m-1, n+1}-\frac{n(1-v) \rho}{2} \zeta_{l, m, n}
\end{aligned}
$$

with the boundary condition $\xi_{0,0,1}=g$ and $\xi_{1,1,0}=p q$. This boundary value problem is uniquely solved by means of the Feynman-Kac formula. That is

$$
\begin{aligned}
& \zeta_{l, m, n} \\
& =g \mathbb{E}_{l, m, n, 0}\left[\exp \left\{-\frac{\rho(1-v)}{2} \int_{0}^{\tau_{v}} c_{v}(u) d u\right\},\left(a_{v}\left(\tau_{v}\right), b_{v}\left(\tau_{v}\right), c_{v}\left(\tau_{v}\right)\right)=(0,0,1)\right] \\
& +p q \mathbb{E}_{l, m, n, 0}\left[\exp \left\{-\frac{\rho(1-v)}{2} \int_{0}^{\tau_{v}} c_{v}(u) d u\right\},\left(a_{v}\left(\tau_{v}\right), b_{v}\left(\tau_{v}\right), c_{v}\left(\tau_{v}\right)\right)=(1,1,0)\right] .
\end{aligned}
$$


On the other hand we have

$$
\begin{aligned}
\mathbb{E}_{l, m, n, 0}\left[p^{a(\tau)} q^{b(\tau)} g^{c(\tau)} v^{s(\tau)}\right]= & g \mathbb{E}_{l, m, n, 0}\left[v^{s(\tau)},(a(\tau), b(\tau), c(\tau))=(0,0,1)\right] \\
& +p q \mathbb{E}_{l, m, n, 0}\left[v^{s(\tau)},(a(\tau), b(\tau), c(\tau))=(1,1,0)\right] .
\end{aligned}
$$

Thus the probability generating functions of the number of recombination events on ancestral lineages of an ARG until a sample has common ancestor at both of the two loci with the given state in which the sample path absorbed are

$$
\begin{aligned}
& \mathbb{E}_{l, m, n, 0}\left[v^{s(\tau)},(a(\tau), b(\tau), c(\tau))=(0,0,1)\right] \\
& =\mathbb{E}_{l, m, n, 0}\left[\exp \left\{-\frac{\rho(1-v)}{2} \int_{0}^{\tau_{v}} c_{v}(u) d u\right\},\left(a_{v}\left(\tau_{v}\right), b_{v}\left(\tau_{v}\right), c_{v}\left(\tau_{v}\right)\right)=(0,0,1)\right]
\end{aligned}
$$

and

$$
\begin{aligned}
& \mathbb{E}_{l, m, n, 0}\left[v^{s(\tau)},(a(\tau), b(\tau), c(\tau))=(1,1,0)\right] \\
& =\mathbb{E}_{l, m, n, 0}\left[\exp \left\{-\frac{\rho(1-v)}{2} \int_{0}^{\tau_{v}} c_{v}(u) d u\right\},\left(a_{v}\left(\tau_{v}\right), b_{v}\left(\tau_{v}\right), c_{v}\left(\tau_{v}\right)\right)=(1,1,0)\right]
\end{aligned}
$$

Lemma 5.6 follows as the summation of these two probability generating functions.

Corollary 5.7. The expected number of recombination events on an ancestral lineages of an $A R G$ until a sample has a common ancestor at both of the two loci is

$$
\begin{aligned}
\mathbb{E}_{l, m, n, 0}[s(\tau)] & =\frac{\rho}{2} \mathbb{E}_{l, m, n, 0}\left[\int_{0}^{\tau} c(u) d u\right] \\
& =\frac{\rho}{2} \sum_{\left(l^{\prime}, m^{\prime}, n^{\prime}\right) \in \mathbb{Z}_{+}^{3} \backslash\{\mathbf{0}, \mathcal{S}\}} \int_{0}^{\infty} k \mathbb{P}_{l, m, n, 0}\left[(a(u), b(u), c(u))=\left(l^{\prime}, m^{\prime}, n^{\prime}\right)\right] d u
\end{aligned}
$$

Proof. Let $T_{l^{\prime}, m^{\prime}, n^{\prime}}$ be the sojourn time of a sample path of the process of the numbers of ancestral lineages in a section of an ARG of a sample $(l, m, n)$ stays at a state $\left(l^{\prime}, m^{\prime}, n^{\prime}\right) \notin$ 
$\mathcal{S}$. Then

$$
\begin{aligned}
& \mathbb{E}_{l, m, n, 0}\left[\int_{0}^{\tau} c(u) d u\right]=\sum_{\left(l^{\prime}, m^{\prime}, n^{\prime}\right) \in \mathbb{Z}_{+}^{3} \backslash\{\mathbf{0}, \mathcal{S}\}} n^{\prime} \mathbb{E}_{l, m, n, 0}\left[T_{l^{\prime}, m^{\prime}, n^{\prime}}\right] \\
& =\sum_{\left(l^{\prime}, m^{\prime}, n^{\prime}\right) \in \mathbb{Z}_{+}^{3} \backslash\{\mathbf{0}, \mathcal{S}\}} n^{\prime} \mathbb{E}_{l, m, n, 0}\left[\int_{0}^{\infty} I_{\left(l^{\prime}, m^{\prime}, n^{\prime}\right)}(a(t), b(t), c(t))\right] \\
& =\sum_{\left(l^{\prime}, m^{\prime}, n^{\prime}\right) \in \mathbb{Z}_{+}^{3} \backslash\{\mathbf{0}, \mathcal{S}\}} n^{\prime} \int_{0}^{\infty} \mathbb{P}_{l, m, n, 0}\left[(a(u), b(u), c(u))=\left(l^{\prime}, m^{\prime}, n^{\prime}\right)\right] d u,
\end{aligned}
$$

where the last equality follows by Fubini's theorem.

Remark 5.8. A recursion of $\mathbb{E}_{l, m, n, 0}[s(\tau)]$ is given by Theorem 6 of [7].

Corollary 5.9. The probability that no recombination events occur on an ARG until a sample has common ancestor at both of the two loci is

$$
\mathbb{P}_{l, m, n, 0}[s(\tau)=0]=\mathbb{E}_{l, m, n, 0}\left[\exp \left\{-\frac{\rho}{2} \int_{0}^{\tau_{0}} c_{0}(u) d u\right\}\right]
$$

Theorem 5.10. The probability that no recombination events occur on an ARG of a sample $(0,0, n)$ until the sample has common ancestor at both of the two loci is

$$
\frac{(n-1) !}{(\rho+1)_{n-1}}
$$

Proof. By (3.1) we see that $a_{0}(t)=b_{0}(t)=0$ for all $t$ and the marginal process $\left\{c_{0}(t) ; t \geq\right.$ $0\}$ is a death process with death rate $i(i-1) / 2$ when $c_{0}(t)=i$. Let a hitting time be $\gamma=\inf \{s \geq 0 ; a(s)=n-1\}$. By Corollary 5.9,

$$
\begin{aligned}
\mathbb{P}_{0,0, n, 0}[s(\tau)=0] & =\mathbb{E}_{0,0, n, 0}\left[\exp \left\{-\frac{\rho}{2} \int_{0}^{\tau_{0}} c_{0}(u) d u\right\}\right] \\
& =\mathbb{E}_{0,0, n, 0}\left\{\mathbb{E}\left[\exp \left\{-\frac{\rho}{2} \int_{0}^{\tau_{0}} c_{0}(u) d u\right\} \mid \gamma\right]\right\} \\
& =\mathbb{E}_{0,0, n, 0}\left\{\mathbb{E}_{0,0, n-1,0}\left[\exp \left\{-\frac{\rho}{2} \int_{0}^{\tau_{0}} c_{0}(u) d u\right\}\right] e^{-\frac{n \rho}{2} \gamma}\right\} \\
& =\mathbb{E}_{0,0, n-1,0}\left[\exp \left\{-\frac{\rho}{2} \int_{0}^{\tau_{0}} c_{0}(u) d u\right\}\right] \frac{n-1}{n-1+\rho},
\end{aligned}
$$


where the third equality follows by the strong Markov property, with the boundary condition

$$
\mathbb{E}_{0,0,1,0}\left[\exp \left\{-\frac{\rho}{2} \int_{0}^{\tau_{0}} c_{0}(u) d u\right\}\right]=1 .
$$

The recursion solved immediately and we get Theorem 5.10 .

Theorem 5.11. The probability that no recombination events occur on an ARG of a sample $(n, 0,1)$ until the sample has common ancestor at both of the two loci is

$$
\prod_{i=0}^{n} \frac{i(i+1)}{i(i+1)+\rho} \text {. }
$$

Proof. By (3.1) we see that $b_{0}(t)=0$ and $c_{0}(t)=1$ for all $t$, and the marginal process $\left\{a_{0}(t) ; t \geq 0\right\}$ is a death process with death rate $i(i+1) / 2$ when $a_{0}(t)=i$. By Corollary 5.9 , we have

$$
\mathbb{P}_{n, 0,1}[s(\tau)=0]=\mathbb{E}_{n}\left[e^{-\frac{\rho}{2} \tau_{0}}\right]
$$

The expression follows by a similar argument as used in Proof of Theorem 5.10,

Finally, let us consider the limit $\rho \rightarrow \infty$. For the purpose we introduce two processes. The one is a diffusion process $\left\{x_{\infty}(t), y_{\infty}(t) ; t \geq 0\right\}$ in $[0,1]^{2}$ with a generator

$$
\mathcal{L}_{\infty}=\frac{x(1-x)}{2} \frac{\partial^{2}}{\partial x^{2}}+\frac{y(1-y)}{2} \frac{\partial^{2}}{\partial y^{2}}
$$

and $\left(x_{\infty}(0), y_{\infty}(0)\right)=(p, q)$. The other is a Markov chain $\left\{a_{\infty}(t), b_{\infty}(t) ; t \geq 0\right\}$ in $\mathbb{Z}_{+}^{2} \backslash \mathbf{0}$ whose transition rates are:

$$
(a, b) \rightarrow \begin{cases}(a-1, b), & a(a-1) / 2, \\ (a, b-1), & b(b-1) / 2 .\end{cases}
$$

and $\left(a_{\infty}(0), b_{\infty}(0)\right)=(l, m)$. Let $\tau_{\infty}=\left\{s \geq 0 ;\left(a_{\infty}(s), b_{\infty}(s)\right) \in\{(1,0),(0,1),(1,1)\}\right\}$.

Theorem 5.12 (Theorem 1 and 2 of [4]). If $\left(x_{\infty}(0), y_{\infty}(0)\right)=(p, q)$, then $\{x(t), y(t) ; t \geq$ $0\}$ converges weakly in $C\left([0, \infty),[0,1]^{2}\right)$ to $\left\{x_{\infty}(t), y_{\infty}(t) ; t \geq 0\right\}$, and $\left\{z(t)-d e^{-\frac{\rho}{2} t} ; t \geq 0\right\}$ converges weakly in $C([0, \infty), \mathbb{R})$ to the zero process in $\mathbb{R}$ as $\rho \rightarrow \infty$. The two function spaces are given the topology of uniform convergence on compact intervals. 
Corollary 5.13. The probability generating function of the distribution of the numbers of ancestral lineages in a section of an $A R G$ of a sample $(a(0), b(0), c(0))=(l, m, n)$ has a limit

$$
\mathbb{E}_{l, m, n}\left[p^{a(t)} q^{b(t)} g^{c(t)}\right] \rightarrow \mathbb{E}_{l+n}\left[p^{a_{\infty}(t)}\right] \mathbb{E}_{m+n}\left[q^{b_{\infty}(t)}\right], \quad \rho \rightarrow \infty
$$

Proof. From Lemma 3.1 we have

$$
\begin{aligned}
\mathbb{E}_{l, m, n}\left[p^{a(t)} q^{b(t)} g^{c(t)}\right]= & \mathbb{E}_{p, q, g}\left[x(t)^{l+n} y(t)^{m+n}\right] \\
& +\sum_{i=1}^{n} \frac{n !}{(n-i) ! i !} \mathbb{E}_{p, q, g}\left[x(t)^{l+n-i} y(t)^{m+n-i} z(t)^{i}\right] .
\end{aligned}
$$

It follows from Theorem 5.12 and Lebesgue's convergence theorem that

$$
\mathbb{E}_{p, q, g}\left[x(t)^{l+n-i} y(t)^{m+n-i} z(t)^{i}\right] \leq \mathbb{E}_{p, q, g}\left[z(t)^{i}\right] \rightarrow 0 \quad \rho \rightarrow \infty
$$

for $t>0, i=1,2, \ldots, n$, while

$$
\mathbb{E}_{p, q, g}\left[x(t)^{l+n} y(t)^{m+n}\right] \rightarrow \mathbb{E}_{p}\left[x_{\infty}(t)^{l+n}\right] \mathbb{E}_{q}\left[y_{\infty}(t)^{m+n}\right]=\mathbb{E}_{l+n}\left[p^{a_{\infty}(t)}\right] \mathbb{E}_{m+n}\left[q^{b_{\infty}(t)}\right] .
$$

as $\rho \rightarrow \infty$. The last equality is a result of the duality between $\left\{x_{\infty}(t) ; t \geq 0\right\}$ and $\left\{a_{\infty}(t) ; t \geq 0\right\}$, and between $\left\{y_{\infty}(t) ; t \geq 0\right\}$ and $\left\{b_{\infty}(t) ; t \geq 0\right\}$.

Corollary 5.13 shows that all $A B$ gametes in a sample instantaneously split into a pair $A-$ and $-B$ gametes in the limit $\rho \rightarrow \infty$. Therefore the length of $\mathcal{C}$ lineages in an ARG goes to zero in this limit.

Theorem 5.14. The expected lengths of $\mathcal{C}$ lineages and whole lineages of an $A R G$ of a sample $(l, m, n)$ until the sample has common ancestor at both of the two loci are

$$
\mathbb{E}_{l, m, n}\left[\int_{0}^{\tau} c(u) d u\right] \rightarrow \frac{2}{\rho} \mathbb{E}_{l, m}\left[\int_{0}^{\tau_{\infty}} a_{\infty}(u) b_{\infty}(u) d u\right]+\frac{2 n}{\rho},
$$

and

$$
\mathbb{E}_{l, m, n}\left[\int_{0}^{\tau}(a(u)+b(u)+c(u)) d u\right] \rightarrow \mathbb{E}_{l, m}\left[\int_{0}^{\tau_{\infty}}\left(a_{\infty}(u)+b_{\infty}(u)\right) d u\right],
$$

respectively. 
Proof. Let $\eta_{l, m, n}=\lim _{\rho \rightarrow \infty} \mathbb{E}_{l, m, n, 0}[s(\tau)=0]$ and $\lambda_{l, m}=\eta_{l, m, 0}$. For $(l, m, n) \in \mathbb{Z}_{+}^{3} \backslash \mathbf{0}$ we have $\eta_{l, m, n}=n+\lambda_{l+n, m+n}$ for $n \geq 1$ and

$$
0=l m-\frac{l(l-1)+m(m-1)}{2} \lambda_{l, m}+\frac{l(l-1)}{2} \lambda_{l-1, m}+\frac{m(m-1)}{2} \lambda_{l, m-1},
$$

with the boundary condition $\lambda_{1,0}=\lambda_{0,1}=\lambda_{1,1}=0$. This boundary value problem is uniquely solved by means of the Feynman-Kac formula. That is

$$
\lambda_{l, m}=\mathbb{E}_{l, m}\left[\int_{0}^{\tau_{\infty}} a_{\infty}(u) b_{\infty}(u) d u\right]
$$

From Corollary 5.7, we have (5.4). Let

$$
\eta_{l, m, n}^{\prime}=\lim _{\rho \rightarrow \infty} \mathbb{E}_{l, m, n, 0}\left[\int_{0}^{\tau_{\infty}}(a(u)+b(u)+c(u)) d u\right]
$$

and $\lambda_{l, m}^{\prime}=\eta_{l, m, 0}^{\prime}$. For $(l, m, n) \in \mathbb{Z}_{+}^{3} \backslash \mathbf{0}$ we have $\eta_{l, m, n}^{\prime}=\lambda_{l, m}^{\prime}$ for $n \geq 1$ and

$$
0=l+m-\frac{l(l-1)+m(m-1)}{2} \lambda_{l, m}^{\prime}+\frac{l(l-1)}{2} \lambda_{l-1, m}^{\prime}+\frac{m(m-1)}{2} \lambda_{l, m-1}^{\prime},
$$

with the boundary condition $\lambda_{1,0}^{\prime}=\lambda_{0,1}^{\prime}=\lambda_{1,1}^{\prime}=0$. This boundary problem is also solved and we have (5.5).

\section{REFERENCES}

[1] Bailey, W. N. (1935). Generalised Hypergeometric Series. Cambridge University Press, Cambridge.

[2] Crow, J. F. And Kimura, M. (1971). Introduction to Population Genetics Theory. Harper and low, New York.

[3] ErdéLy, A. ed. (1953). Higher Transcendental Functions, Vol. I. McGrow-Hill, New York.

[4] Etheir, S. N. (1979). A limit theorem for two-locus diffusion models in population genetics. J. Appl. Probab. 16, 402-408.

[5] Etheir, S. N. And Griffiths, R. C. (1990a). The neutral two-locus model as a measure-valued diffusion. Adv. Appl. Prob. 22, 773-786.

[6] Etheir, S. N. And Griffiths, R. C. (1990b). On the two-locus sampling distribution. J. Math. Biol.

29, 131-159. 
[7] Griffiths, R. C. (1991). The two-locus ancestral graph. In Selected proceedings of the symposium on applied probability, Sheffield, 1989. ed. I.V. Basawa and R.L. Taylor. IMS Lecture notes-monograph series, 18, pp. 100-117.

[8] Griffiths, R. C. And Tavar'E, R. C. (1994). Sampling theory for neutral alleles in a varying environment. Phil. Trans. R. Soc. Lond. B 344, 403-410.

[9] Hudson, R. R. And Kaplan, N. L. (1985). Statistical properties of the number of recombination events in the history of a sample of DNA sequences. Genetics 111, 147-164.

[10] Kimura, M.(1955). Solution of a process of random genetic drift with a continuous model. Proc. Acad. Natul. Sci. USA 41, 144-150.

[11] Kimura, M.(1955). Stochastic process and distribution of gene frequencies under natural selection. Cold Spring Harbor Symposia on Quantitative Biology 20, 33-53.

[12] Kingman, J. F. C. (1982). The coalescent. Stoch. Proc. Appl. 13, 235-248.

[13] Krone, S. M. And Neuhauser, C. (1997). Ancestral process with selection. Theor. Popul. Biol. 51, 210-237.

[14] Liggett, T. M. (1985). Interacting Particle Systems. Springer-Verlag, Berlin.

[15] Littler, R. A. (1972). Multidimensional stochastic models in genetics. Ph.D. Thesis. Monash University.

[16] MalÉcot, G. (1948) Les Mathematiques de l'hérédité. Masson et Cie, Éditeurs.

[17] Mano, S. (2005). Random Genetic Drift and Gamete Frequency. Genetics 171, 2043-2050.

[18] Mano, S. (2009a). Duality, ancestral and diffusion processes in models with selection. Theor. Popul. Biol. 75, 164-175.

[19] Mano, S. (2009b). Ancestral process with bias in gene conversion. arXiv:0907.1127

[20] Оhta, T. and Kimura, M. (1969). Linkage disequilibrium due to random genetic drift. Genet. Res. 13, $47-55$.

[21] Shiga, T. (1981). Diffusion processes in population genetics. J. Math. Kyoto Univ. 21, $133-151$.

[22] TavarÉ, S. (2004). Ancestral inference in population genetics. In Lectures on Probability Theory and Statistics. Ecole d'Eté de Probabilités de Saint-Flour XXXI-2001. ed. J. Picard. Lecture Notes in Mathematics, 1837, 1-188. Springer-Verlag, New York. 